\title{
Design and Synthesis of Peptide Derivatives Act as DNA Binding Agent and Discovery of Potent Carbonic Anhydrase Inhibitors Using Docking Studies
}

\author{
A. A. El-Henawy, Mona S. Kadah* and H.S.A. Nassar \\ Chemistry Department, Faculty of Science and *Chemistry \\ Department, Faculty of Science (For Girls), Al-Azhar \\ University, Cairo, Egypt.
}

\begin{abstract}
EW peptide series of 2-(2-oxo-2H benzo[h] chromen-4-yl) acetyl derivatives 3-5, 15-20 and their corresponding methyl esters 6-14 were synthesized. Structures of the synthesized products were characterized by correct elemental analysis, IR, Mass and ${ }^{1} \mathrm{H}-\mathrm{NMR}$ spectroscopy. DNA binding activities were performed. Some synthesized compounds showed high binding affinity against DNA. Virtual screening using molecular docking studies of the synthesized compounds were performed, the molecular docking results indicate that, most of the synthesized compounds are more suitable inhibitors against carbonic anhydrase isozyme (CA) than reference drug.
\end{abstract}

Study of conformational changes of DNA (deoxyribonucleic acid) by ligand binding are a pharmacologic target of many drugs ${ }^{(1-5)}$. The structural changes of DNA based on the interaction of small molecular weight ligands with DNA have attracted attention in the medicinal design of anticancer and anti-AIDS drugs ${ }^{(6,7)}$. Some chromen-2-one (coumarine) derivatives have been reported to possess antiSARS agent ${ }^{(8)}$, antioxidant properties $^{(9)}$, anti-fungal ${ }^{(10)}$, anti-bacterial ${ }^{(11)}$, antiinflammatory $^{(12)}$, antitumor ${ }^{(13)}$, inhibitors of pancreatic cholesterol ${ }^{(14)}$, estrogen receptor ${ }^{(15)}$ and treatment of Alzheimer diseases ${ }^{(16)}$. In addition, many drugs consist of amino acid moieties as; AG7088 (which was developed by Pfizer and used for the treatment of rhinovirus, which can cause the common cold), which contain phenylalanine moiety ${ }^{(17)}$.

There are a variety of mechanisms for the anticancer activity and the most famous mechanism is through the inhibition of carbonic anhydrase isozymes ${ }^{(18-22)}$. In brief, the CA is a family of metalloenzymes involved in the catalysis of an important physiological reaction: the hydration of $\mathrm{CO}_{2}$ to bicarbonate and a proton $\left(\mathrm{CO}_{2}+\mathrm{H}_{2} \mathrm{O} \mathrm{HCO}_{3}^{-}+\mathrm{H}^{+}\right)$, where bicarbonate is necessary to synthesis of nucleotides and other cell components such as membrane lipids. The synthesized compounds may decrease supplying of bicarbonate. Based on the foregoing, the present study undertakes synthesis of peptide series and their methyl esters of 2 (2-oxo-2H-benzo[h]-chromen-4-yl)acetyl derivatives, with hope to obtain new candidate(s) as antitumor agents . 


\section{Result and Discussion}

\section{Chemistry}

The formation of dipeptides 3-5 was achieved by the reaction of 2-(2-oxo- $2 \mathrm{H}-$ benzo[h]chromen-4-yl) acetyl chloride 2 with different type of amino acids (Scheme 1). Also, 3-5 were reacted with thionyl chloride (molar ratio) in methanol to give the corresponding dipeptide methyl esters 6-8 (Scheme 1). Tripeptide methyl esters 9-14 were prepared by the reaction compounds 3-5 with different amino acid methyl esters in presence of tetrahydrofuran and few drops of triethyl amine using carbodiimide technique ${ }^{(18)}$ (Scheme 1). When compounds 14-19 were treated with sodium hydroxide $(0.01 \mathrm{~N})$ was converted into corresponding tripeptides $15-20$. The results of chemical analyses of the synthesized compounds were summarized in Tables 1-3

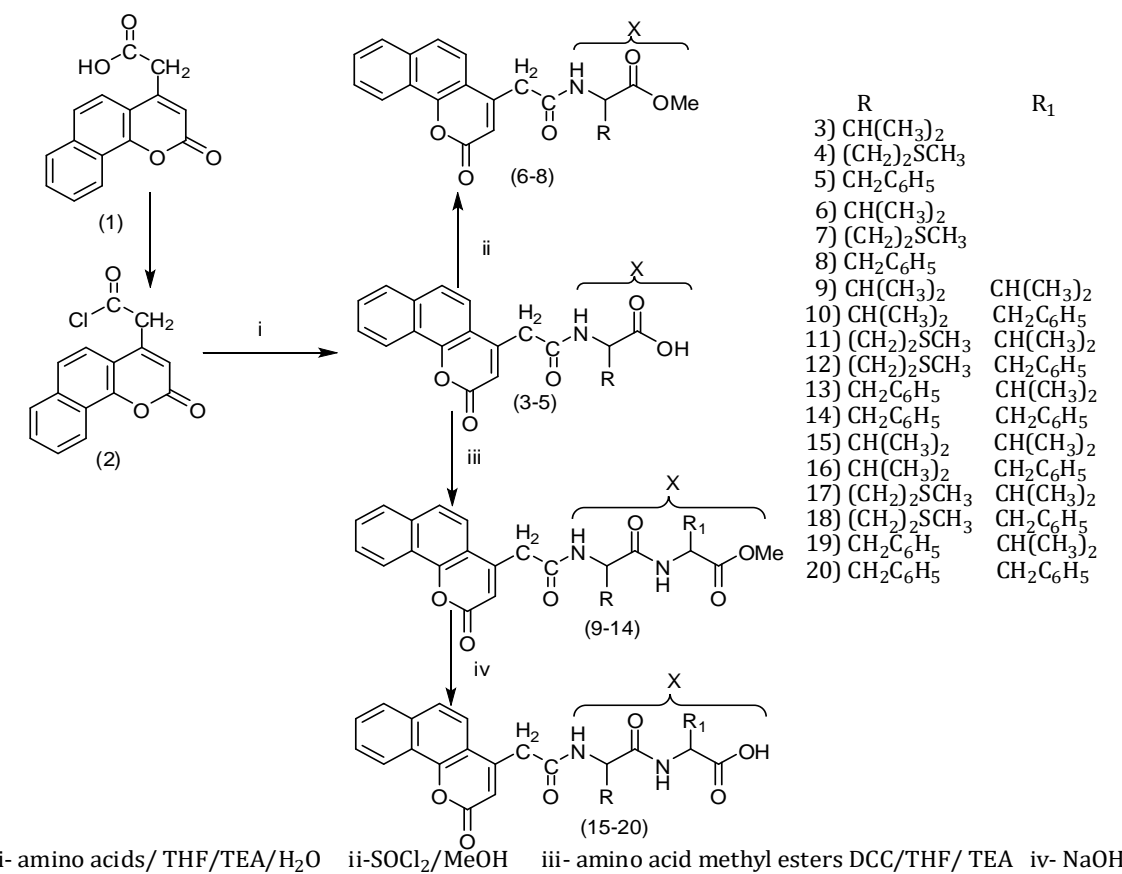

Scheme 1

\section{Biological Activity}

DNA as an affinity probe for evaluation of biologically active compounds

DNA binding assay

The mechanism of several antitumor compounds and antitumor antibiotics depends on their interaction with DNA. In this work, the antitumor activities of the newly synthesized compounds were determined using DNA binding assay and methyl green. DNA displacement assay ${ }^{(23,24)}$. In this method, a fixed amount of the ligand is spotted on the RP-18 TLC plates, followed by addition of known amount of

Egypt. J. Chem. 53, No.2 (2010) 
DNA on the same spot. The plate was then developed and the position of unbound DNA was determined by spraying the plates with anisaldehyde reagent. The free DNA was detected as a blue spot (Rf, $\mathrm{MeOH}-\mathrm{H}_{2} \mathrm{O}, 8: 2$ ) on RP-18 TLC. It was demonstrated that, when DNA was mixed with compounds known to interact with it, e.g. ethidium bromide, the complex was retained at the origin. Compounds with high binding affinity to DNA remained on the base line or migrated for a very short distance, while compounds with poor binding affinity did not cause DNA to be retained at the origin ${ }^{(23)}$.

TABLE 1. Physical data for the compounds (3-20).

\begin{tabular}{|c|c|c|c|c|c|c|c|c|c|c|}
\hline \multirow[t]{2}{*}{ Compd. } & \multirow[t]{2}{*}{$\mathbf{X}$} & \multirow[t]{2}{*}{$\begin{array}{l}\text { Yield } \\
\%\end{array}$} & \multirow[t]{2}{*}{$\underset{{ }^{\circ} \mathrm{C}}{\mathrm{M} . \mathrm{P}}$} & \multirow[t]{2}{*}{ Color } & \multirow[t]{2}{*}[\alpha]{$_{D}^{20}$} & \multirow[t]{2}{*}{$\mathbf{R}_{\mathbf{F}}$} & \multirow[t]{2}{*}{$\begin{array}{c}\text { Molecular Formula } \\
\text { (M.Wt.) }\end{array}$} & \multicolumn{3}{|c|}{$\begin{array}{c}\text { Elemental Analysis } \\
\text { Calculated/Found }\end{array}$} \\
\hline & & & & & & & & C & $H$ & $N$ \\
\hline 3 & L-Val & 76 & $182-84$ & yellow & +77 & 0.53 & $\begin{array}{c}\mathrm{C}_{20} \mathrm{H}_{19} \mathrm{NO}_{5} \\
(353)\end{array}$ & $\begin{array}{l}67.98 \\
67.98\end{array}$ & $\begin{array}{l}5.42 \\
5.40\end{array}$ & $\begin{array}{l}3.96 \\
3.92\end{array}$ \\
\hline 4 & L-Met & 82 & $175-77$ & yellow & +86 & 0.80 & $\begin{array}{c}\mathrm{C}_{20} \mathrm{H}_{19} \mathrm{NO}_{5} \mathrm{~S} \\
(385)\end{array}$ & $\begin{array}{l}62.32 \\
62.31\end{array}$ & $\begin{array}{l}4.97 \\
4.98\end{array}$ & $\begin{array}{l}3.63 \\
3.61\end{array}$ \\
\hline 5 & L-Phe & 75 & $191-93$ & yellow & +119 & 0.87 & $\begin{array}{c}\mathrm{C}_{24} \mathrm{H}_{19} \mathrm{NO}_{5} \\
(401)\end{array}$ & $\begin{array}{l}71.81 \\
71.80\end{array}$ & $\begin{array}{l}4.77 \\
4.77\end{array}$ & $\begin{array}{l}3.49 \\
3.50\end{array}$ \\
\hline 6 & $\begin{array}{l}\text { L-Val } \\
\text {-OMe }\end{array}$ & 69 & $200-202$ & brown & +80 & 0.86 & $\begin{array}{c}\mathrm{C}_{21} \mathrm{H}_{21} \mathrm{NO}_{5} \\
(367)\end{array}$ & $\begin{array}{l}68.65 \\
68.63\end{array}$ & $\begin{array}{l}5.76 \\
5.74\end{array}$ & $\begin{array}{l}3.81 \\
3.82\end{array}$ \\
\hline 7 & $\begin{array}{l}\text { L-Met } \\
\text {-OMe }\end{array}$ & 65 & $210-12$ & Red & +98 & 0.89 & $\begin{array}{c}\mathrm{C}_{21} \mathrm{H}_{21} \mathrm{NO}_{5} \mathrm{~S} \\
\quad(399)\end{array}$ & $\begin{array}{l}63.14 \\
63.15\end{array}$ & $\begin{array}{l}5.30 \\
5.30\end{array}$ & $\begin{array}{l}3.51 \\
3.50\end{array}$ \\
\hline 8 & $\begin{array}{l}\text { L-Phe } \\
\text {-OMe }\end{array}$ & 78 & $193-95$ & Red & +116 & 0.60 & $\begin{array}{c}\mathrm{C}_{25} \mathrm{H}_{21} \mathrm{NO}_{5} \\
(415)\end{array}$ & $\begin{array}{l}72.28 \\
72.28\end{array}$ & $\begin{array}{l}5.10 \\
5.10\end{array}$ & $\begin{array}{l}3.37 \\
3.35\end{array}$ \\
\hline 9 & $\begin{array}{l}\text { L-Val-L- } \\
\text { Val-OMe }\end{array}$ & 86 & $166-68$ & Red & +134 & 0.68 & $\begin{array}{c}\mathrm{C}_{26} \mathrm{H}_{30} \mathrm{~N}_{2} \mathrm{O}_{6} \\
(466)\end{array}$ & $\begin{array}{l}66.94 \\
66.91\end{array}$ & $\begin{array}{l}6.48 \\
6.45\end{array}$ & $\begin{array}{l}6.00 \\
6.01\end{array}$ \\
\hline 10 & $\begin{array}{l}\text { L-Val-L- } \\
\text { Phe-OMe }\end{array}$ & 80 & 207-209 & Red & +128 & 0.64 & $\begin{array}{l}\mathrm{C}_{30} \mathrm{H}_{30} \mathrm{~N}_{2} \mathrm{O}_{6} \\
\quad(514)\end{array}$ & $\begin{array}{l}70.02 \\
70.00\end{array}$ & $\begin{array}{l}5.88 \\
5.88\end{array}$ & $\begin{array}{l}5.44 \\
5.46\end{array}$ \\
\hline 11 & $\begin{array}{l}\text { L-Met-L- } \\
\text { Val-OMe }\end{array}$ & 75 & $179-81$ & Red & +120 & 0.69 & $\begin{array}{c}\mathrm{C}_{26} \mathrm{H}_{30} \mathrm{~N}_{2} \mathrm{O}_{6} \mathrm{~S} \\
(498)\end{array}$ & $\begin{array}{l}62.63 \\
62.63\end{array}$ & $\begin{array}{l}6.06 \\
6.03\end{array}$ & $\begin{array}{l}5.62 \\
5.63\end{array}$ \\
\hline 12 & $\begin{array}{l}\text { L-Met-L- } \\
\text { Phe-OMe }\end{array}$ & 78 & 206-208 & Red & +109 & 0.65 & $\begin{array}{c}\mathrm{C}_{30} \mathrm{H}_{30} \mathrm{~N}_{2} \mathrm{O}_{6} \mathrm{~S} \\
\quad(546)\end{array}$ & $\begin{array}{l}65.92 \\
65.90\end{array}$ & $\begin{array}{l}5.53 \\
5.52\end{array}$ & $\begin{array}{l}5.12 \\
5.13\end{array}$ \\
\hline 13 & $\begin{array}{l}\text { L-Phe-L- } \\
\text { Val-OMe }\end{array}$ & 80 & $197-99$ & Red & +103 & 0.67 & $\begin{array}{c}\mathrm{C}_{30} \mathrm{H}_{30} \mathrm{~N}_{2} \mathrm{O}_{6} \\
(514)\end{array}$ & $\begin{array}{l}70.02 \\
70.00\end{array}$ & $\begin{array}{l}5.88 \\
5.88\end{array}$ & $\begin{array}{l}5.44 \\
5.44\end{array}$ \\
\hline 14 & $\begin{array}{l}\text { L-phe-L- } \\
\text { Phe-OMe }\end{array}$ & 75 & $210-12$ & orange & +85 & 0.79 & $\begin{array}{l}\mathrm{C}_{34} \mathrm{H}_{30} \mathrm{~N}_{2} \mathrm{O}_{6} \\
(562)\end{array}$ & $\begin{array}{l}72.58 \\
72.56\end{array}$ & $\begin{array}{l}5.37 \\
5.34\end{array}$ & $\begin{array}{l}4.98 \\
5.00\end{array}$ \\
\hline 15 & $\begin{array}{l}\text { L-Val- } \\
\text { L-Val }\end{array}$ & 85 & $160-62$ & Red & +98 & 0.84 & $\begin{array}{c}\mathrm{C}_{25} \mathrm{H}_{28} \mathrm{~N}_{2} \mathrm{O}_{6} \\
(452)\end{array}$ & $\begin{array}{l}66.36 \\
66.34\end{array}$ & $\begin{array}{l}6.19 \\
6.22\end{array}$ & $\begin{array}{l}6.19 \\
6.20\end{array}$ \\
\hline 16 & $\begin{array}{l}\text { L-Val- } \\
\text { L-Phe }\end{array}$ & 70 & $192-94$ & Red & +125 & 0.91 & $\begin{array}{c}\mathrm{C}_{29} \mathrm{H}_{28} \mathrm{~N}_{2} \mathrm{O}_{6} \\
(500)\end{array}$ & $\begin{array}{l}69.59 \\
69.60\end{array}$ & $\begin{array}{l}5.60 \\
5.62\end{array}$ & $\begin{array}{l}5.60 \\
5.60\end{array}$ \\
\hline 17 & $\begin{array}{l}\text { L-Met- } \\
\text { L-Val }\end{array}$ & 78 & $216-18$ & Red & +142 & 0.82 & $\begin{array}{c}\mathrm{C}_{25} \mathrm{H}_{28} \mathrm{~N}_{2} \mathrm{O}_{6} \mathrm{~S} \\
(484)\end{array}$ & $\begin{array}{l}61.97 \\
61.97\end{array}$ & $\begin{array}{l}5.78 \\
5.80\end{array}$ & $\begin{array}{l}5.78 \\
5.78\end{array}$ \\
\hline 18 & $\begin{array}{l}\text { L-Met- } \\
\text { L-Phe }\end{array}$ & 77 & $200-202$ & Red & +162 & 0.77 & $\begin{array}{c}\mathrm{C}_{29} \mathrm{H}_{28} \mathrm{~N}_{2} \mathrm{O}_{6} \mathrm{~S} \\
(532)\end{array}$ & $\begin{array}{l}65.40 \\
65.39\end{array}$ & $\begin{array}{l}5.26 \\
5.30\end{array}$ & $\begin{array}{l}5.26 \\
5.36\end{array}$ \\
\hline 19 & $\begin{array}{l}\text { L-Phe- } \\
\text { L-Val }\end{array}$ & 70 & 209-11 & Red & +112 & 0.94 & $\begin{array}{c}\mathrm{C}_{29} \mathrm{H}_{28} \mathrm{~N}_{2} \mathrm{O}_{6} \\
(500)\end{array}$ & $\begin{array}{l}69.59 \\
69.60\end{array}$ & $\begin{array}{l}5.60 \\
5.64\end{array}$ & $\begin{array}{l}5.60 \\
5.62\end{array}$ \\
\hline 20 & $\begin{array}{l}\text { L-phe- } \\
\text { L-phe }\end{array}$ & 68 & $187-89$ & Red & +117 & 0.81 & $\begin{array}{c}\mathrm{C}_{33} \mathrm{H}_{28} \mathrm{~N}_{2} \mathrm{O}_{6} \\
(548)\end{array}$ & $\begin{array}{l}72.25 \\
72.22\end{array}$ & $\begin{array}{l}5.11 \\
5.14\end{array}$ & $\begin{array}{l}5.11 \\
5.12\end{array}$ \\
\hline
\end{tabular}


Methyl green-DNA displacement assay

Methyl green reversibly binds polymerized DNA forming a stable complex at neutral $\mathrm{pH}^{(24)}$. The maximum absorption for the DNA-methyl green complex is $642-645 \mathrm{~nm}$. This colorimetric assay was used to measure the displacement of methyl green with DNA by compounds, which have the ability to bind with DNA. The degree of displacement was determined spectrophotometrically by measuring the change in the initial absorbance of the DNA-methyl green solution in the presence of reference compound.

TABLE 2. IR and mass spectra of the synthesized products.

\begin{tabular}{|c|c|c|}
\hline Compd. & IR $\left(v_{\max } \mathbf{c m}^{-1}\right)$ Selected bands & MS $\left[\mathbf{M}^{+}\right]$ \\
\hline 3 & $\begin{array}{l}3400(\mathrm{OH}) ; 3331(\mathrm{NH}) ; 3090\left(\mathrm{CH} \text {-aromatic), 2969,2931 }\left(\mathrm{CH}_{3^{-}}\right.\right. \\
\text {valine); 1725,1733 (CO). }\end{array}$ & $(353 ; 22 \%)$ \\
\hline 4 & $3380(\mathrm{OH}) ; 3122(\mathrm{NH}) ; 3090(\mathrm{CH}$-aromatic), 1716,1690 (CO). & $(385 ; 43 \%)$ \\
\hline 5 & $3410(\mathrm{OH}) ; 3250(\mathrm{NH}) ; 3076(\mathrm{CH}$-aromatic), 1718,1670 (CO). & $(401 ; 56 \%)$ \\
\hline 6 & $3200(\mathrm{NH}), \quad 1720,1700(\mathrm{CO})$ & $(367 ; 22 \%)$ \\
\hline 7 & $3229(\mathrm{NH}), 1726,1685 \quad(\mathrm{CO})$ & $(399 ; 12 \%)$ \\
\hline 8 & $3233(\mathrm{NH}), 1733,1648$ (CO). & $(415 ; 42 \%)$ \\
\hline 9 & $3300,3125(\mathrm{NH}) ; 1720-1678 \quad(\mathrm{CO})$ & $(466 ; 82 \%)$ \\
\hline 10 & $3250,3131(\mathrm{NH}) ; 1725-1680 \quad(\mathrm{CO})$ & $(514 ; 79 \%)$ \\
\hline 11 & $3173,3115(\mathrm{NH}) ; 1744-1637 \quad(\mathrm{CO})$ & $(498 ; 40 \%)$ \\
\hline 12 & $3173,3115(\mathrm{NH}) ; 1748-1637 \quad(\mathrm{CO})$ & $(546 ; 47 \%)$ \\
\hline 13 & $3185,3122(\mathrm{NH}) ; 1750-1699(\mathrm{CO})$. & $(514 ; 24 \%)$ \\
\hline 14 & $3331,3285(\mathrm{NH}) ; 1714-1690(\mathrm{CO})$ & $(562 ; 100 \%)$ \\
\hline 15 & 3342, $3331(\mathrm{OH}, \mathrm{NH}) ; 1718-1690(\mathrm{CO})$. & $(453 ; 84 \%)$ \\
\hline 16 & $3422 \mathrm{~cm}^{-1}(\mathrm{OH}) ; 3332,3250(\mathrm{NH})$ 1750-1702 (CO). & $(500 ; 64 \%)$ \\
\hline 17 & 3358,3116 (OH,NH); 1718-1696 (CO). & $(484 ; 57 \%)$ \\
\hline 18 & $3382(\mathrm{OH}) ; 3338,3241(\mathrm{NH})$ 1718-1652 (CO). & $(532 ; 27 \%)$ \\
\hline 19 & $3414(\mathrm{OH}) ; 3356,3230(\mathrm{NH}) 1718-1672(\mathrm{CO})$ & $(500 ; 5 \%)$ \\
\hline 20 & $3416(\mathrm{OH}) ; 3345,3210(\mathrm{NH})$ 1706-1690 (CO). & $(548 ; 36 \%)$ \\
\hline
\end{tabular}


TABLE 3. ${ }^{1} \mathrm{H}$ NMR results for the synthesized compounds.

\begin{tabular}{|c|c|}
\hline Comp. & ${ }^{1}$ HNMR $\delta$ \\
\hline 3 & $\begin{array}{l}\left.10.33\left(\mathrm{~s}, 1 \mathrm{H}, \mathrm{OH}-\mathrm{COOH}, \mathrm{D}_{2} \mathrm{O} \text {-exchangeable }\right]\right), 8.13(\mathrm{~d}, 1 \mathrm{H}, \mathrm{NH}, \mathrm{D} 2 \mathrm{O}- \\
\text { exchangeable }]), 7.54-6.89(\mathrm{~m}, 6 \mathrm{H}, 2 \mathrm{Ar}-\mathrm{H}), 6.65(\mathrm{~s}, 1 \mathrm{H}, \mathrm{CH}-\mathrm{h} \text { - } \\
\text { exoro }), 3.78(\mathrm{~d}, 1 \mathrm{H} \text {, } \\
\left.\mathrm{CH}-\mathrm{CH}-\mathrm{CH}(\mathrm{CH} 3)_{2}\right), 3.01\left(\mathrm{~s}, 2 \mathrm{H}, \mathrm{CH}_{2} \mathrm{CO}\right), 2.94\left(\mathrm{~m}, 1 \mathrm{H}, \mathrm{CH}-\mathrm{CH}(\mathrm{CH} 3)_{2}, 1.74(\mathrm{~d},\right. \\
\left.6 \mathrm{H}, 2\left(\mathrm{CH}_{3}\right)_{2}\right) \text {. }\end{array}$ \\
\hline 5 & $\begin{array}{l}10.63\left(\mathrm{~s}, 1 \mathrm{H}, \mathrm{OH}-\text { carboxylic, } \mathrm{D}_{2} \mathrm{O}-\text { exchangeable }\right), 8.52\left(\mathrm{~d}, 1 \mathrm{H}, \mathrm{NH}, \mathrm{D}_{2} \mathrm{O}-\right. \\
\text { exchangeable }]), \quad 8.0-7.1(\mathrm{~m}, 11 \mathrm{H}, 3 \mathrm{Ar}-\mathrm{H}), \quad 6.52(\mathrm{~s}, 1 \mathrm{H}, \mathrm{CH} \text {-hetero }), 4.09(\mathrm{t} \text {, } \\
\left.1 \mathrm{H}, \mathrm{CH}-\mathrm{CH}_{2}\right), 2.96\left(\mathrm{~s}, 2 \mathrm{H}, \mathrm{CH}_{2}-\mathrm{CO}\right), 2.82\left(\mathrm{~d}, 2 \mathrm{H}, \mathrm{CH}_{2} \mathrm{Ph}\right) .\end{array}$ \\
\hline 7 & $\begin{array}{l}\left.\text { 8.37(d, 1H,NH, } \mathrm{D}_{2} \mathrm{O} \text {-exchangeable }\right), 7.92-7.12(\mathrm{~m}, 5 \mathrm{H}, 2 \mathrm{Ar}-\mathrm{H}), 6.32(\mathrm{~s}, 1 \mathrm{H} \text {, } \\
\mathrm{CH}-\text { hetero }), 4.05\left(\mathrm{t}, 1 \mathrm{H}, \mathrm{CH}-\left(\mathrm{CH}_{2}\right)_{2}\right), 3.89\left(\mathrm{~s}, 3 \mathrm{H}, \mathrm{CH}_{3}-\mathrm{OCH}_{3}\right), 3.01\left(\mathrm{~s}, 2 \mathrm{H}, \mathrm{CH}_{2-}\right. \\
\mathrm{CO}), 2.69-2.63\left(\mathrm{t}, 2 \mathrm{H}, \mathrm{CH}_{2}-\mathrm{SCH}_{3}\right), 1.84-1.63\left(\mathrm{~m}, 2 \mathrm{H}, \mathrm{CH}_{2}-\mathrm{CH}_{2}\right), 1.32(\mathrm{~s}, 3 \mathrm{H} \text {, } \\
\left.\mathrm{CH}_{3}\right) \text {. }\end{array}$ \\
\hline 8 & $\begin{array}{l}\left.\text { 8.54(d, } 1 \mathrm{H}, \mathrm{NH}, \mathrm{D}_{2} \mathrm{O}-\text { exchangeable }\right), 7.53-6.86(\mathrm{~m}, 11 \mathrm{H}, 3 \mathrm{Ar}-\mathrm{H}), \\
6.46(\mathrm{~s}, 1 \mathrm{H}, \mathrm{CH}-\text { hetero }), 4.05\left(\mathrm{t}, 1 \mathrm{H}, \mathrm{CH}-\left(\mathrm{CH}_{2}\right)_{2}\right), 3.89\left(\mathrm{~s}, \quad 3 \mathrm{H}, \mathrm{CH}_{3}-\mathrm{OCH}_{3}\right), \\
\left.\text { 2.95(s,2H, } \mathrm{CH}_{2}-\mathrm{CO}\right) \text { and other bands confirmed the structure. }\end{array}$ \\
\hline 10 & $\begin{array}{l}\quad 8.30\left(\mathrm{~d}, 1 \mathrm{H}, \mathrm{NH}, \mathrm{D}_{2} \mathrm{O}-\text {-exchangeable }\right), 7.73-6.74(\mathrm{~m}, 5 \mathrm{H}, 2 \mathrm{Ar}-\mathrm{H}), 6.54(\mathrm{~s}, 1 \mathrm{H}, \\
\mathrm{CH} \text {-hetero }), 5.92\left(\mathrm{~s}, 1 \mathrm{H}, \mathrm{NH}, \mathrm{D}_{2} \mathrm{O}-\text {-exchangeable }\right), 4.05\left(\mathrm{t}, 1 \mathrm{H}, \mathrm{CH}-\mathrm{CH}_{2} \mathrm{Ph}\right), 3.79 \\
\left(\mathrm{~d}, 1 \mathrm{H}, \mathrm{CH}-\mathrm{CH}\left(\mathrm{CH}_{3}\right)_{2}\right), 3.42\left(\mathrm{~s}, 3 \mathrm{H}, \mathrm{CH}_{3}-\mathrm{OCH}_{3}\right), 3.14\left(\mathrm{~d}, 2 \mathrm{H}-\mathrm{CH}_{2} \mathrm{Ph}\right) ; 2.83 \\
\left(\mathrm{~s}, 2 \mathrm{H}, \mathrm{CH}_{2}-\mathrm{CO}\right), 2.31-3.12\left(\mathrm{~m}, 1 \mathrm{H}, \mathrm{CH}-\mathrm{CH}\left(\mathrm{CH}_{3}\right)_{2}\right), 1.43\left(\mathrm{~s}, 6 \mathrm{H}, 2 \mathrm{CH}_{3}-\left(\mathrm{CH}_{3}\right)_{2}\right) .\end{array}$ \\
\hline 11 & $\begin{array}{l}\quad 8.37\left(\mathrm{~d}, 1 \mathrm{H}, \mathrm{NH}, \mathrm{D}_{2} \mathrm{O} \text {-exchangeable }\right), 7.87-7.00(\mathrm{~m}, 6 \mathrm{H}, 2 \mathrm{Ar}-\mathrm{H}), 6.67(\mathrm{~s}, 1 \mathrm{H}, \\
\mathrm{CH}-\text { hetero }), 6.02\left(\mathrm{~s}, 1 \mathrm{H}, \mathrm{NH}, \mathrm{D}_{2} \mathrm{O} \text {-exchangeable }\right), 3.77\left(\mathrm{~s}, 3 \mathrm{H}, \mathrm{CH}_{3}-\mathrm{OCH}_{3}\right), \\
3.63\left(\mathrm{t}, 1 \mathrm{H}, \mathrm{CH}-\left(\mathrm{CH}_{2}\right)_{2} \mathrm{SCH}_{3}\right), 3.24\left(\mathrm{~d}, 1 \mathrm{H}, \mathrm{CH}-\mathrm{CH}\left(\mathrm{CH}_{3}\right)_{2}\right), 2.91\left(\mathrm{~s}, 2 \mathrm{H}, \mathrm{CH}_{2}-\mathrm{CO}\right), \\
2.62-2.55 \quad\left(\mathrm{~m}, \quad 1 \mathrm{H}, \quad \mathrm{CH}-\mathrm{CH}\left(\mathrm{CH}_{3}\right)_{2}\right), 2.26-2.16\left(\mathrm{t}, 2 \mathrm{H}, \mathrm{CH}_{2}-\mathrm{SCH}_{3}\right), \\
1.74\left(\mathrm{~m}, 2 \mathrm{H}, \mathrm{CH}_{2}-\mathrm{CH}_{2} \mathrm{SCH}_{3}\right), 1.64\left(\mathrm{~s}, 3 \mathrm{H}, \mathrm{CH}_{3}-\mathrm{SCH}_{3}\right), 1.34\left(\mathrm{~s}, 6 \mathrm{H}, 2 \mathrm{CH}_{3}-\left(\mathrm{CH}_{3}\right)_{2}\right) .\end{array}$ \\
\hline 13 & $\begin{array}{l}\quad 8.55\left(\mathrm{~d}, 1 \mathrm{H}, \mathrm{NH}, \mathrm{D}_{2} \mathrm{O} \text {-exchangeable }\right), 7.58-6.74(\mathrm{~m}, 6 \mathrm{H}, 2 \mathrm{Ar}-\mathrm{H}), 6.47(\mathrm{~s}, 1 \mathrm{H} \text {, } \\
\mathrm{CH}-\text { hetero }), 5.87\left(\mathrm{~s}, 1 \mathrm{H}, \mathrm{NH}, \mathrm{D}_{2} \mathrm{O}-\text {-exchangeable }\right) 4.28\left(\mathrm{t}, 1 \mathrm{H}, \mathrm{CH}-\mathrm{CH}_{2} \mathrm{Ph}\right), 3.71 \\
\left(\mathrm{~s}, 3 \mathrm{H}, \mathrm{CH}_{3}-\mathrm{OCH}_{3}\right), 3.44\left(\mathrm{t}, 1 \mathrm{H}, \mathrm{CH}-\mathrm{CH}_{2} \mathrm{Ph}\right), 2.74\left(\mathrm{~s}, 2 \mathrm{H}, \mathrm{CH}_{2}-\mathrm{CO}\right), 2.68(\mathrm{~d}, 1 \mathrm{H} \text {, } \\
\mathrm{CH}-\mathrm{Ph}) \text { and other bands confirmed the structure. }\end{array}$ \\
\hline 16 & 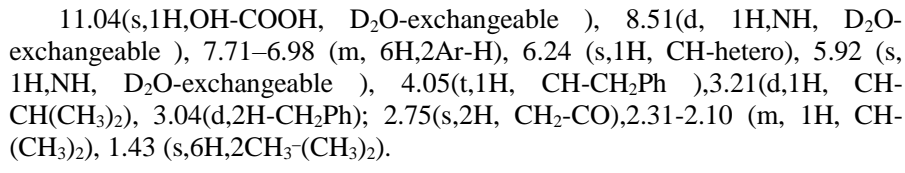 \\
\hline 18 & 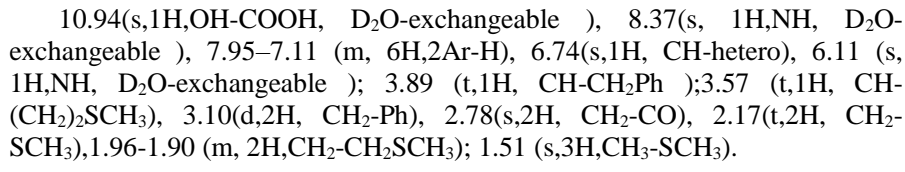 \\
\hline 19 & 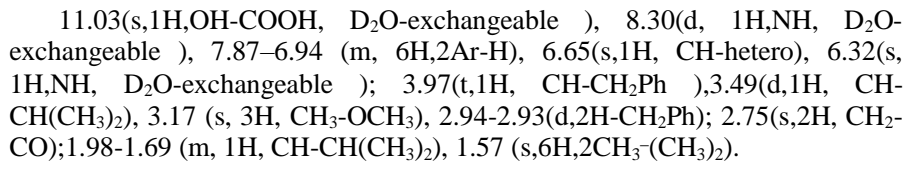 \\
\hline
\end{tabular}


Results from DNA binding assay (Table 4) revealed that, Compounds 4,7,9,13 and 16 showed the highest affinity for DNA, which was demonstrated by retaining the complex at the origin or by migrating for a very short distances, and by measuring $\mathrm{IC}_{50}$ (concentration required for $50 \%$ decrease in the initial absorbance of the DNA/methyl green solution). Compounds 6,10,11 and 17 showed moderate activity while compounds 8,18,19 and 20 showed weak activity. The remaining synthesized compounds showed the lowest affinity for DNA, which was demonstrated by migrating for a very long distance at the origin, represented graphically (Fig.1).

TABLE 4. DNA binding activity of the synthesized compounds 3-20 using methyl green DNA displacements assay.

\begin{tabular}{|c|c|c|c|c|c|}
\hline NO. & $\mathrm{IC}_{50}(\mu g / m l .)^{*}$ & $\mathrm{IC}_{50}(\mu M .)^{* *}$ & NO. & $\mathrm{IC}_{50}(\mu g / m l .)^{*}$ & $\mathrm{IC}_{50}(\mu M .)^{* *}$ \\
\hline 3 & 23 & 65.15 & 4 & 20 & 51.94 \\
\hline 5 & ND & ND & 6 & 21 & 55.58 \\
\hline 7 & 19 & 47.62 & 8 & 82 & 197.59 \\
\hline 9 & 18 & 38.62 & 10 & 37 & 71.99 \\
\hline 11 & 41 & 82.33 & 12 & ND & ND \\
\hline 13 & 12 & 23.35 & 14 & ND & ND \\
\hline 15 & ND & ND & 16 & 15 & 30.00 \\
\hline 17 & 39 & 75.88 & 18 & 57 & 107.14 \\
\hline 19 & 68 & 136 & 20 & 45 & 82.11 \\
\hline Dau. & 29 & 53.13 & & & \\
\hline
\end{tabular}

ND) Not determined (Compounds having IC $_{50}$ value $>100 \mu / \mathrm{ml}$.).

*) $\mathrm{IC}_{50}$ Values: Represented $\mathrm{IC}_{50}$ obtained from three independent determinations required for $50 \%$ decrease in the initial absorbance of DNA-methyl green solution.

**) $\mathrm{IC}_{50}$ values: Concentration required for $50 \%$ decrease in the initial absorbance of DNA-methyl green solution.

Molecular modeling studies

To predict the anti-tumor behavior of the synthesized derivatives 3-20 on a structural basis, automated docking studies were carried out using Molegro virtual docker ${ }^{(25)}$. The scoring functions and hydrogen bonds formed with the surrounding amino acids, which are used to predict their binding modes at the active site of the human carbonic anhydrase II (hCA II). The scoring functions were calculated from minimized ligand protein complexes. The protein-ligand complex was constructed based on the X-ray structure (PDB entry 1G54) bounded with its inhibitor FFB-555 ${ }^{(26)}$. 


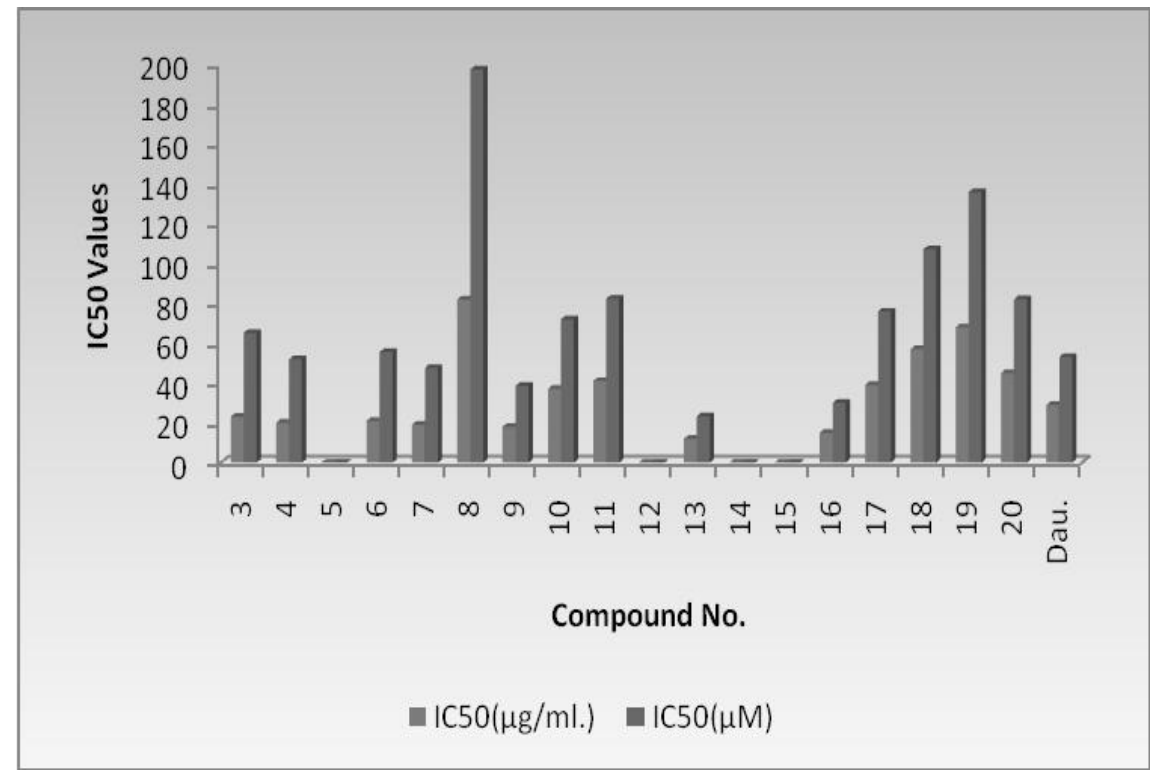

Fig.1. DNA binding activity of compounds (3-20) using methyl green DNA displacements assay as $\mathrm{IC}_{50}$ (concentration required for $50 \%$ decrease in the initial absorbance of the DNA/methyl green solution).

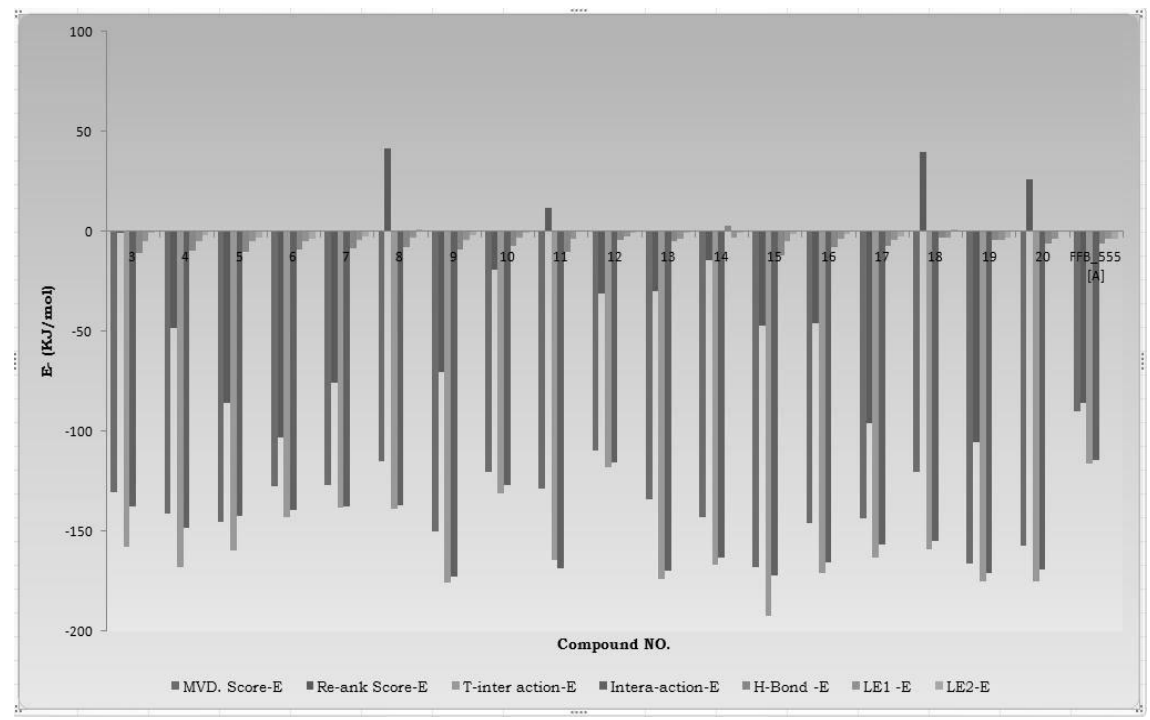

Fig. 2. Showed different scores derived from the MVD docking tools.

Egypt. J. Chem. 53, No.2 (2010) 
From the comparative docking study of the synthesized compounds with (FFB-555) and observe how the compounds bind to the kinase binding site, based on the knowledge of the structure of similar active sites from analysis of the $\mathrm{X}$ ray crystal structure of human carbonic anhydrase II (hCA II) with (E7070) ${ }^{(20)}$. We docked the synthesized compounds 3-20 into the empty binding site of (1G54), with its bound inhibitor (FFB-555), Fig. 3 show binding mode of the original ligand into its binding site, while Fig. $4 \& 5$ show binding modes of compounds 5 and 19 , respectively.

As shown from Tables 5\& 6 and Fig. 3-5, the following results can be drawn:

E7070 (the original ligand) reveals MVD score (-90.03) and form three hydrogen bonds: two hydrogen bond with Thr-199 and one hydrogen bond with His-119 (Fig.3).

Compound 5 gave strong binding affinity with MVD score (-145.517) and form 18 bonds with active binding site: two important bonds with $\mathrm{Zn}$ metal, two other important bonds with Thr-199, three bonds with (Thr-200 anad His-119), one bond with ( Glu-106, His-96 and His-94). (Fig.4).

Also, Compound 19 gave strong binding affinity with MVD score (-166.088) and form eighteen bonds with active binding site: two important bonds with $\mathrm{Zn}$ metal, three other important bonds with Thr-199, three bonds with (Thr-200 anad His-119), two important bonds with (His-119, His 94 and Gln-92 ) and one bond with ( Glu-106, His-96, Asn-67, Asn-64 and His-64).

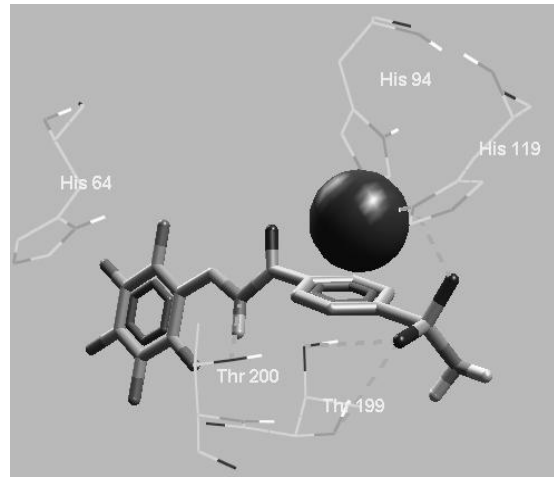

A

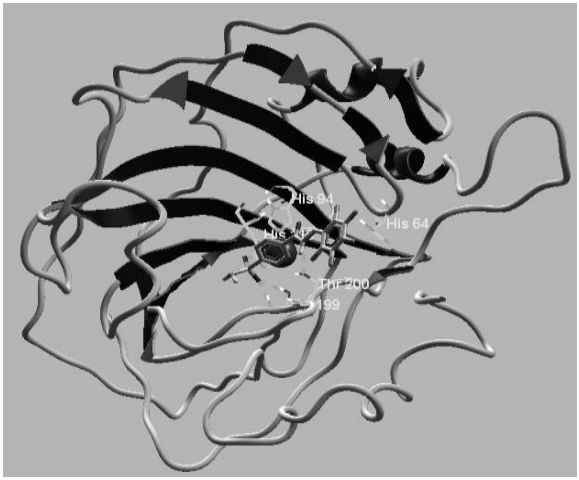

B

Fig.3. A) Interaction between (FFB) and binding site of CA (1G54, PDB code), which Green dot lines represented hydrogen bonding interaction of ligand (E7070) with binding site. Docked compound (E7070) is represented in stick mode, which atoms are colored in dark grey, oxygen in red, nitrogen in blue and sulfur in yellow. Hydrogen atoms of the amino acid residues and ligand were removed to improve clarity.

B) A plot of docked ligand (FFB) in active site where the backbone of protein is shown in flat ribbon. 

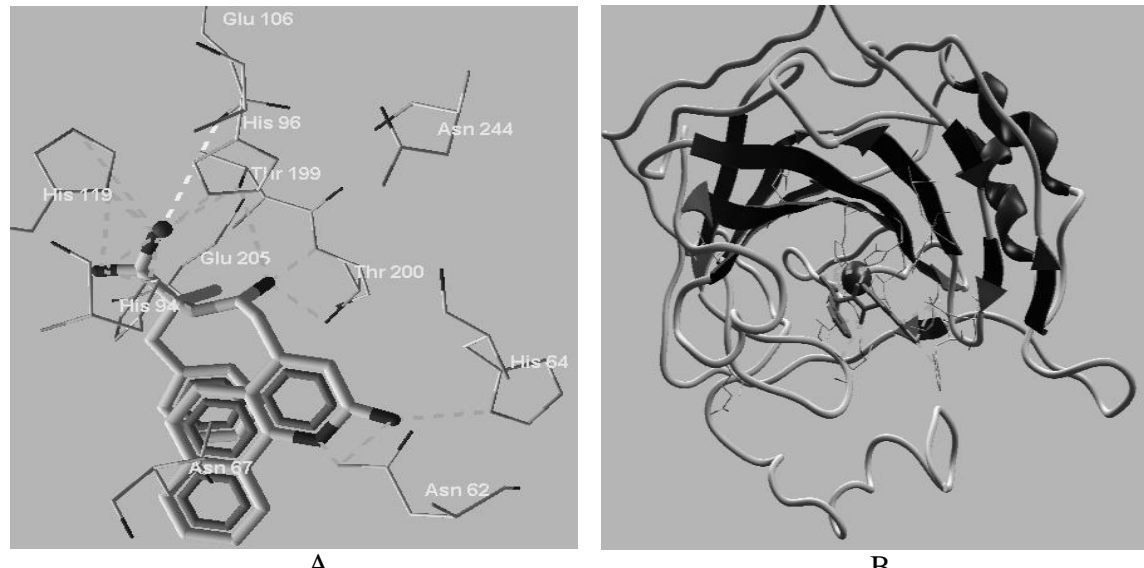

A

B

Fig.4. A) Interaction between ligand (5) and binding site of CA 1G54, PDB code), which green dot lines represented hydrogen bonding interaction of ligand (5) with binding site, yellow dot line represented electrostatic interaction of ligand (5) with binding site. Ligand (5) is represented in stick mode, which atoms are colored in dark grey, oxygen in red, nitrogen in blue and sulfur in yellow. Hydrogen atoms of the amino acid residues and ligand were removed to improve clarity.

B) A plot of docked ligand (5) in active site where the backbone of protein is shown in flat ribbon.

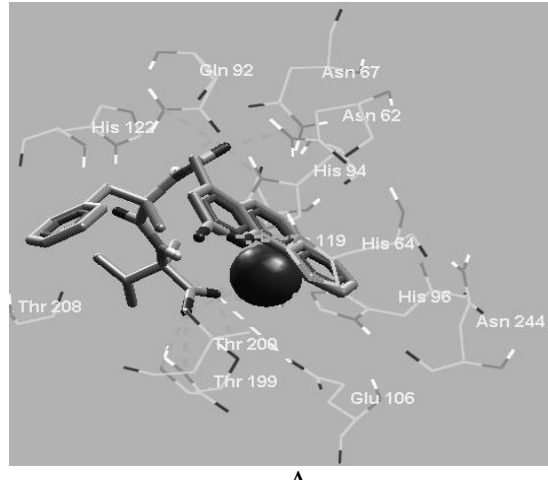

A

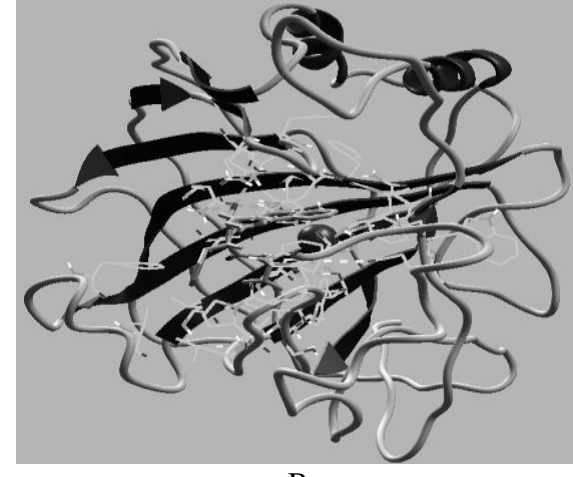

B

Fig. 5. A) Interaction between ligand (19) and binding site of CA (1G54, PDB code), which green dot lines represented hydrogen bonding interaction of ligand (19) with binding site. Ligand (19) is represented in stick mode, which atoms are colored in dark grey, oxygen in red, nitrogen in blue and sulfur in yellow. Hydrogen atoms of the amino acid residues and ligand were removed to improve clarity.

B) A plot of docked ligand (19) in active site where the backbone of protein is shown in flat ribbon 
TABLE 5. Showed different scores derived from the MVD docking tools.

\begin{tabular}{cccccccc}
\hline NO. & $\begin{array}{c}\text { MVD. } \\
\text { Score-E }\end{array}$ & $\begin{array}{c}\text { affinity } \\
\text {-E }\end{array}$ & $\begin{array}{c}\text { T-inter } \\
\text { action- } \\
\text { E }\end{array}$ & $\begin{array}{c}\text { Inter- } \\
\text { action-E }\end{array}$ & $\begin{array}{c}\text { H-Bond } \\
\text {-E }\end{array}$ & $\begin{array}{c}\text { LE1 } \\
-\mathbf{E}\end{array}$ & $\begin{array}{c}\text { LE2 } \\
\mathbf{- E}\end{array}$ \\
\hline $\mathbf{F B B}$ & -90.0384 & -85.8086 & -115.89 & -114.53 & -6.09668 & -3.60154 & -3.43234 \\
$\mathbf{3}$ & -130.326 & -0.38792 & -157.608 & -137.279 & -10.749 & -4.8269 & -0.0143674 \\
$\mathbf{4}$ & -141.259 & -48.099 & -167.859 & -148.502 & -9.81888 & -5.04495 & -1.71782 \\
$\mathbf{5}$ & -145.517 & -85.6385 & -159.809 & -142.487 & -10.1736 & -4.6941 & -2.76253 \\
$\mathbf{6}$ & -127.573 & -103.273 & -142.959 & -139.194 & -9.00793 & -4.55617 & -3.68833 \\
$\mathbf{7}$ & -126.845 & -75.462 & -137.947 & -137.571 & -8.61806 & -4.37397 & -2.60214 \\
$\mathbf{8}$ & -114.609 & 41.6497 & -138.677 & -136.846 & -7.97827 & -2.86522 & 1.04124 \\
$\mathbf{9}$ & -150.096 & -69.941 & -175.71 & -172.752 & -9.06891 & -4.16934 & -1.94281 \\
$\mathbf{1 0}$ & -120.185 & -18.9083 & -130.676 & -126.942 & -7.41885 & -3.00462 & -0.472707 \\
$\mathbf{1 1}$ & -128.714 & 11.852 & -164.444 & -168.641 & -10.3075 & -3.47876 & 0.320324 \\
$\mathbf{1 2}$ & -109.326 & -30.9755 & -117.991 & -115.643 & -4.32672 & -2.66649 & -0.7555 \\
$\mathbf{1 3}$ & -134.024 & -29.6718 & -173.946 & -169.601 & -4.6524 & -3.35061 & -0.741794 \\
$\mathbf{1 4}$ & -143.097 & -14.4901 & -166.506 & -162.8 & 2.79054 & -3.2522 & -0.329321 \\
$\mathbf{1 5}$ & -168.068 & -46.8467 & -192.27 & -172.204 & -11.8771 & -4.80194 & -1.33848 \\
$\mathbf{1 6}$ & -146.016 & -45.5448 & -170.981 & -165.62 & -7.8758 & -3.744 & -1.16782 \\
$\mathbf{1 7}$ & -143.16 & -95.9654 & -162.882 & -156.657 & -7.0596 & -3.97667 & -2.66571 \\
$\mathbf{1 8}$ & -119.972 & 40.0006 & -158.787 & -154.502 & -3.09716 & -2.99929 & 1.00002 \\
$\mathbf{1 9}$ & -166.088 & -105.62 & -174.787 & -170.656 & -4.22561 & -4.25866 & -2.70821 \\
$\mathbf{2 0}$ & -157.309 & 26.3942 & -174.897 & -169.203 & -5.7745 & -3.65834 & 0.613818 \\
\hline & & & & & & &
\end{tabular}

Where: MVD Score $(\mathrm{KJ} / \mathrm{mol})$ :Energy score used during docking .

Re-rank Score $(\mathrm{Kj} / \mathrm{mol})$ : The re-ranking score.

$\mathrm{H}$. Bond Enrgy $(\mathrm{Kj} / \mathrm{mol})$ :Hydrogen bonding energy between protein and ligand.

T.interaction $(\mathrm{Kj} / \mathrm{mol})$ : The total interaction energy between the pose and the target molecule.

Intera-action affinity $(\mathrm{Kj} / \mathrm{mol})$ : The total interaction energy between the pose and the protein.

LE1(Kj/mol):MolDock Score divided by Heavy Atoms count.

LE2 (Kcal/mol):Rerank Score divided by Heavy Atoms count.

In conclusion, most of the synthesized compounds, showed significant activity, and will be considered as a guide for further future synthetic design . In addition, the docking study of the compounds showed that, the most synthesized compounds act as CA inhibitors more than (E7070). SO, the further modification of these compounds may be promising candidates for clinically useful drug agents. 
TABLE 6. Interaction between ligand and amino acid residues derived from MVD docking tools.

\begin{tabular}{|c|c|c|c|}
\hline $\begin{array}{l}\text { Cpd. } \\
\text { NO. }\end{array}$ & $\begin{array}{c}\text { NO. of } \mathrm{H} \text {. } \\
\text { Bond }\end{array}$ & $\begin{array}{c}\text { Involved group of amino } \\
\text { acid }\end{array}$ & Involved atom of ligand \\
\hline \multirow[t]{4}{*}{ FBB } & 4 & (ND1)His-119...H & O-24(ㅁSO) \\
\hline & & (N)Thr-199...H & 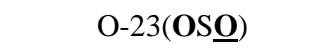 \\
\hline & & (OG1)Thr-199...H & \\
\hline & & (OG1)Thr-200...H & $\mathrm{N}-7(\mathrm{NH})$ \\
\hline \multirow[t]{12}{*}{3} & 13 & $\mathrm{Zn} . . . \mathrm{H}$ & $\mathrm{O}-24(\mathrm{CO} \underline{\mathrm{O}} \mathrm{H})$ \\
\hline & & (N)Thr-200...H & \\
\hline & & (OG1)Thr-200...H & \\
\hline & & (N)Thr-199...H & \\
\hline & & (OG1)Thr-199...H & \\
\hline & & $\mathrm{Zn} . . . \mathrm{H}$ & $\mathrm{O}-23(\mathrm{C} \underline{\mathbf{O}} \mathrm{OH})$ \\
\hline & & (OG1)Thr-199...H & \\
\hline & & (ND1)His119...(2)H & \\
\hline & & (OE1)Glu106...E & \\
\hline & & (NE2)His96...H & \\
\hline & & (NE2)His94...H & \\
\hline & & (OG1)Thr-200...H & O-1(Pyrane) \\
\hline \multirow[t]{12}{*}{4} & 12 & $\mathrm{Zn} \ldots \mathrm{H}$ & $\mathrm{O}-24(\mathrm{CO} \underline{\mathbf{O}} \mathrm{H})$ \\
\hline & & (OG1)Thr-199...H & \\
\hline & & (ND1)His119...(2)H & \\
\hline & & (NE2)His96...H & \\
\hline & & (NE2)His94...H & \\
\hline & & $\mathrm{Zn} \ldots \mathrm{H}$ & $\mathrm{O}-23(\mathrm{C} \underline{\mathbf{O}} \mathrm{OH})$ \\
\hline & & (OG1)Thr-200...H & \\
\hline & & (N)Thr-200...H & \\
\hline & & (OG1)Thr-200...H & \\
\hline & & (OG1)Thr-199...H & \\
\hline & & (NE2)His64...H & O-1(O-Pyrane) \\
\hline & & (ND1)His64...H & \\
\hline \multirow[t]{3}{*}{5} & 18 & $\mathrm{Zn} \ldots \mathrm{H}$ & $\mathrm{O}-24(\mathrm{CO} \underline{\mathbf{O}} \mathrm{H})$ \\
\hline & & (N)Thr-200...H & \\
\hline & & (ND1)His-119...H & \\
\hline
\end{tabular}


TABLE 6. Contd.

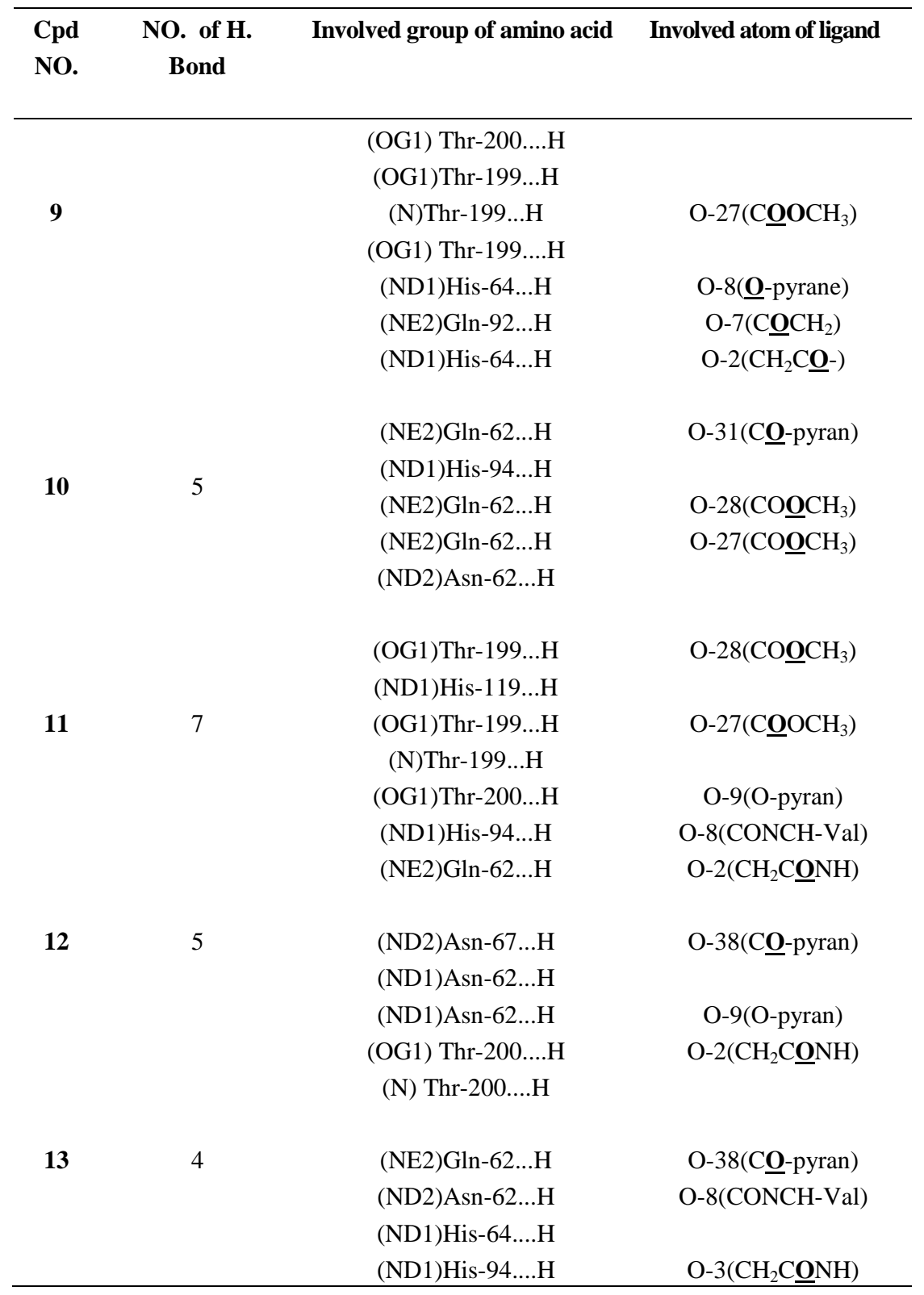


TABLE 6. Contd.

\begin{tabular}{|c|c|c|c|}
\hline $\begin{array}{l}\text { Cpd } \\
\text { NO. }\end{array}$ & NO. of H. Bond & Involved group of amino acid & $\begin{array}{c}\text { Involved atom of } \\
\text { ligand }\end{array}$ \\
\hline & & (NE2)His-94...H & \\
\hline & & $\mathrm{Zn} \ldots \mathrm{H}$ & $\mathrm{O}-23(\mathrm{C} \underline{\mathbf{O O H}})$ \\
\hline & & (OG1)Thr-199...H & \\
\hline & & (NE2)His-119... H & \\
\hline & & (ND1)His-119...(2)H & \\
\hline & & (OE1)Glu-106...E & \\
\hline & & (NE2)His-96...H & \\
\hline & & (NE2)His-94...H & \\
\hline & & (OG1)Thr-199...H & O-18(CㅁNH) \\
\hline & & (N)Thr-200...H & \\
\hline & & (OG1)Thr-200...H & \\
\hline \multirow[t]{6}{*}{6} & 8 & $(\mathrm{OH}) \mathrm{Tyr}-7 \ldots \mathrm{H}$ & $\mathrm{O}-24(\mathrm{CO} \underline{\mathbf{O}} \mathrm{H})$ \\
\hline & & (ND2)Asn-67...H & O-18(CㅁNH) \\
\hline & & (ND2)Asn-62...H & \\
\hline & & (OG1)Thr-200...H & O-16(Cㅁ-pyrane) \\
\hline & & (N)Thr-200...H & \\
\hline & & (OG1)Thr-199...H & \\
\hline \multirow[t]{3}{*}{7} & 3 & (NE2)Gln-92...H & $\mathrm{O}-18\left(\mathrm{CO}^{\mathrm{O}} \mathrm{CH}_{3}\right)$ \\
\hline & & (ND1)His-64...H & O-16(Cㅁ-pyrane) \\
\hline & & (ND1)His-64...H & O-1(ㅇ-pyrane) \\
\hline \multirow[t]{5}{*}{8} & 5 & (ND2)Asn-62...H & O-18(CㅁNH) \\
\hline & & (ND1)His-64...H & O-7(CQ-pyrane) \\
\hline & & (ND2)Asn-62...H & \\
\hline & & (ND1)His-94...H & \\
\hline & & (ND2)Asn-62...H & $\mathrm{O}-2(\mathrm{C} \underline{\mathbf{O}}-\mathrm{NH})$ \\
\hline \multirow[t]{3}{*}{9} & 10 & (ND1)His-64...H & O-31(C足-pyrane) \\
\hline & & (N)Thr-200...H & $\mathrm{O}-28\left(\mathrm{CO}^{\mathrm{O}} \mathrm{CH}_{3}\right)$ \\
\hline & & $(\mathrm{N})$ Thr-199...H & \\
\hline
\end{tabular}


TABLE 6. Contd.

\begin{tabular}{|c|c|c|c|}
\hline $\begin{array}{l}\text { Cpd } \\
\text { NO. }\end{array}$ & NO. of H. Bond & $\begin{array}{l}\text { Involved group of } \\
\text { amino acid }\end{array}$ & Involved atom of ligand \\
\hline \multirow[t]{4}{*}{14} & 4 & (NE2)Gln-92....H & 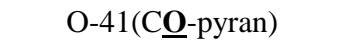 \\
\hline & & (ND2)Asn-62...H & $\mathrm{O}-8\left(\mathrm{CH}_{2} \mathrm{C} \underline{\mathbf{O} N H}-\mathrm{Phe}\right)$ \\
\hline & & (OG1)Thr-200...H & $\mathrm{N}-4\left(\mathrm{CH}_{2} \mathrm{CON} H-\mathrm{Phe}\right)$ \\
\hline & & (OG1)Thr-200...H & $\mathrm{O}-3\left(\mathrm{CH}_{2} \mathrm{CON} H-\mathrm{Phe}\right)$ \\
\hline \multirow{15}{*}{15} & 15 & (ND2)Asn-62...H & O-33(Cㅁ-pyran) \\
\hline & 15 & $\mathrm{Zn} \ldots \mathrm{H}$ & $\mathrm{O}-28(\mathrm{CO} \underline{\mathbf{O}} \mathrm{H})$ \\
\hline & & (OG1)Thr-200...H & \\
\hline & & (N)Thr-200...H & \\
\hline & & (OG1)Thr-199...H & \\
\hline & & (N)Thr-199...H & \\
\hline & & $\mathrm{O}-28(\mathrm{CO} \underline{\mathrm{O}} \mathrm{H})$ & $\mathrm{O}-27(\mathrm{C} \underline{\mathbf{O}} \mathrm{OH})$ \\
\hline & & (OG1)Thr-199...H & \\
\hline & & (OE1)Glu-106...E & \\
\hline & & (NE2)His-96....H & \\
\hline & & (ND1)His-119....(2)H & \\
\hline & & (NE2)His-96....H & \\
\hline & & (ND2)Asn-62...H & O-8(O-pyran) \\
\hline & & (NE2)Gln-92....H & $\mathrm{O}-2\left(\mathrm{CH}_{2} \mathrm{C} \underline{\mathrm{O} N H}\right)$ \\
\hline & & (ND1)His-96... (2)H & O-37(Cㅇ-pyran) \\
\hline 16 & 3 & (NE2)Gln-92... H & $\mathrm{O}-27(\mathrm{C} \underline{\mathbf{O O H}})$ \\
\hline \multirow[t]{8}{*}{17} & 9 & (ND1)His-64... (2)H & O-28(COㅁH) \\
\hline & & (ND2)Asn-62... H & \\
\hline & & (OG1)Thr-200...H & O-27(CㅁOH) \\
\hline & & (ND1)His-64... (2)H & \\
\hline & & (OG1)Thr-200...H & N-23(NH-Val) \\
\hline & & (ND1)His-94... H & O-7(CㅁNH-Val) \\
\hline & & (NE2)Gln-92... H & O-2(C $\underline{\text { ONH}}$-Met) \\
\hline & & (ND1)His-94... H & O-7(CO \\
\hline
\end{tabular}


TABLE 6. Contd.

\begin{tabular}{|c|c|c|c|}
\hline $\begin{array}{l}\text { Cpd } \\
\text { NO. }\end{array}$ & NO. of H. Bond & $\begin{array}{l}\text { Involved group of } \\
\text { amino acid }\end{array}$ & $\begin{array}{c}\text { Involved atom of } \\
\text { ligand }\end{array}$ \\
\hline 18 & 3 & $\begin{array}{l}\text { (ND1)His-64...H } \\
\text { (NE2)Gln-92... H } \\
\text { (OG1)Thr-199...H }\end{array}$ & 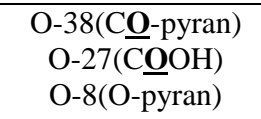 \\
\hline 19 & 18 & $\begin{array}{c}\text { (ND)His-64...H } \\
\text { Zn... H } \\
\text { (N)Thr-200...H } \\
\text { (OG1)Thr-199... } \\
\text { (N)Thr-199...H } \\
\text { Zn... H } \\
\text { (OE1)Glu-106... } \\
\text { (OG1)Thr-199...H } \\
\text { (NE2)His-96...H } \\
\text { (NE2)His-94...H } \\
\text { (ND1)His-119.... (2)H } \\
\text { (ND2)His-119...H } \\
\text { (ND2)Asn-64...H } \\
\text { (NE2)Gln-92...H } \\
\text { (ND1)His-94...H } \\
\text { (NE2)Gln-92...H } \\
\text { (NE2)Asn-67...H }\end{array}$ & $\begin{array}{c}\mathrm{O}-8(\mathrm{O}-\mathrm{pyran}) \\
\mathrm{N}-3\left(\mathrm{CH}_{2} \mathrm{CON} \mathbf{H}\right) \\
\mathrm{O}-2\left(\mathrm{CH}_{2} \mathrm{CON} \mathrm{H}\right) \\
\text { (NE2)Gln-92....H } \\
\text { (NE2)Asn-67....H }\end{array}$ \\
\hline 20 & 9 & $\begin{array}{c}\text { (OG1)Thr-200...H } \\
\text { (N)Thr-200...H } \\
\text { (OG1)Thr-199...H } \\
\text { (N)Thr-199...H } \\
\text { (ND1)His-64...H } \\
\text { (ND2)Asn-62...H } \\
\text { (ND1)His-94...H } \\
\text { (ND2)Asn-67...H } \\
\text { (N)Thr-199...H }\end{array}$ & $\begin{array}{c}\mathrm{O}-7(\mathrm{C} \underline{\mathbf{O}} \mathrm{HCOOH}) \\
\mathrm{O}-2\left(\mathrm{CH}_{2} \mathrm{C} \underline{\mathbf{O} N H}\right) \\
\text { O-8(O-pyran })\end{array}$ \\
\hline
\end{tabular}

\section{Expermintal}

Melting points were taken on a Griffin melting point apparatus and are uncorrected. Electronic spectra were recorded on a UV-UNICAM 2001 spectrophotometer using $10 \mathrm{~mm}$ pass length quartz cells at room temperature. IR spectra were recorded on a Perkin-Elmer spectrophotometer model 1430 as potassium bromide pellets and frequencies are reported in $\mathrm{cm}^{-1} .{ }^{1} \mathrm{H}$ NMR spectra were observed on a Varian Genini-300 MHz spectrometer and chemical shifts $(\delta)$ are in ppm. Mass spectra were recorded on a mass spectrometer HP model MSQPLO00EX (Shimadzu) at $70 \mathrm{eV}$. Elemental analyses $(\mathrm{C}, \mathrm{H}, \mathrm{N})$ were carried out at the 
Microanalytical Center of Cairo University, Giza, Egypt. Biological activities were carried out at the Biogenetic Engineering Center, Molecular Biology Unit, Al-Azhar University, Nasr City, Egypt.

Synthesis of 2-(1-oxo-1H-benzo[h]-chromen-4-yl)acetyl dipeptids (3-5)

General procedures

The desired amino acids (1.5 equiv) were dissolved in water $(25 \mathrm{ml})$ and THF $(15 \mathrm{ml})$ mixture, triethylamine $(2 \mathrm{ml})$ was added, followed by portionwise addition of 2-(1-oxo-1H-benzo- [h]-chromen-4-yl)acetyl chloride (2; 1 equiv) during $30 \mathrm{~min}$. Temperature of the reaction mixture was kept at $10^{\circ} \mathrm{C}$ during the addition. Stirring was continued for $2 \mathrm{hr}$ at $20^{\circ} \mathrm{C}$. Tetrahydrofuran was removed by concentration of the reaction mixture under reduced pressure; water $(30 \mathrm{ml})$ was added and acidified with $2 \mathrm{M} \mathrm{HCL}$ to $\mathrm{pH}$ 5. The crude products were filtered and recrystallized from Methanol. All the products (3-5) were chromatographically homogeneous by iodine and benzidine development.

Synthesis 2-(1-oxo-1H-benzo[h]-chromen-4-yl)acetyl dipeptide methyl esters ( 6-8)

General procedures

To a suspension of coupling reaction products ( $3-5 ; 1$ equiv) in absolute methanol $(150 \mathrm{ml})$ was cooled to $-10^{\circ} \mathrm{C}$ and pure thionyl chlorid (1 equiv) was added dropwise during one hour. The reaction mixture was stirred for an additional $34 \mathrm{hr}$ at room temperature, then kept overnight then the solvent was removed by vacuum distillation. The residual solid material was recrystallized from methanol. All the products (6-8) were chromatographically homogeneous by iodine and benzidine development.

Preparation of 2-(1-oxo-1H-benzo[h]-chromen-4-yl)acetyl tripeptid methyl esters (9-14)

General procedures

To a solution of amino acid methyl ester hydrochloride (1.5 equiv) was dissolved in THF, triethylamine $(2 \mathrm{ml})$ was added, the solution was stirred at $20^{\circ} \mathrm{C}$ for $30 \mathrm{~min}$ and cooled to $\mathrm{O}^{\circ} \mathrm{C}$, dipeptide (3-5;1equiv) in THF (50 ml) and DCC ( 1 equiv) were added to the above mixture. The reaction mixture was stirred for $6 \mathrm{hr}$ at $0^{\circ} \mathrm{C}$ and for another $12 \mathrm{hr}$ at room temperature. The crude material is diluted with EtOAc and washed with sat. aq. $\mathrm{Na}_{2} \mathrm{CO}_{3}(\times 2)$ and brine $(\times 1)$, dried over sodium sulfate, evaporated, and purified by methanol to give the desired products. Products 9-14 were to be chromatogramphically homogeneous by iodine and benzidine development.

Preparation of 2-(2-(1-oxo-1H-benzo[h]-chromen-4-yl)acetyl tripeptid methyl esters (15-20)

General procedures

Tripeptide metyl esters $(9-14 ; 0.001$ mole) were added to a solution of $0.1-\mathrm{N}$ $\mathrm{NaOH}$. The mixture was stirred for $1 \mathrm{hr}$ at $\left(100{ }^{\circ} \mathrm{C}\right)$. The reaction mixture was allowed to cool, then acidified with $1 \mathrm{~N}-\mathrm{HCl}$ to $\mathrm{pH}=5$. The crude products $(15-20)$ were separated, filtered and purified by recrystallization from Methanol. The

Egypt. J. Chem. 53, No.2 (2010) 
products $(15-20)$ were to be chromatogramphically homogeneous by iodine and benzidine development.

\section{Biological Screening}

\section{Evaluation of the degree of DNA binding}

DNA binding assay on TLC plates

Analyses of the DNA binding affinity of the tested compounds were predeveloped first using methanol-water $(8: 2)$. The tested compounds were then applied $(5 \mathrm{mg} / \mathrm{ml}$ in methanol) at the origin, followed by the spotting of DNA (1 $\mathrm{mg} / \mathrm{ml}$ in methanol-water mixture $(8: 2)$ at the same positions at the origin. Daunomycin was used as a positive control. After complete spotting, the plates were developed with the same solvent system and the positions of DNA were visualized by spraying the plates with anisaldehyde, which produces a blue colour with DNA. The intensity of the colour was proportional to the quantity of DNA added to the plate.

Colorimetric assay for the degree of DNA binding

DNAlmethyl green complex $(20 \mathrm{mg}$ ) was suspended in $100 \mathrm{ml}$ of $0.05 \mathrm{M}$ tris$\mathrm{HCl}$ buffer ( $\mathrm{pH} 7.5$ ) containing $7.5 \mathrm{mM} \mathrm{MgSO}_{4}$ and stirred at $37{ }^{\circ} \mathrm{C}$ with a magnetic stirrer for $24 \mathrm{hr}$. The calculated amounts of samples were placed in Eppendorf tubes and $(200 \mu \mathrm{l})$ of the DNA-methyl green solution was added to each tube. The samples were incubated in dark at room temperature and after $24 \mathrm{hr}$. the final absorbance of each sample was determined at (642-645) $\mathrm{nm}$. The results were recorded in form of the $\mathrm{IC}_{50}$ for each compound, which is the sample concentration required to produce $50 \%$ decrease in the initial absorbance of the DNA-methyl green complex. The molar concentration required for $50 \%$ decrease in the initial absorbance of the DNA-methyl green complex was calculated and the results are given in Table 4.

\section{Molecular modelling study}

Generation of ligand and enzyme structures

Docking study was carried out for the target compounds into (hCAII) using Molegro virtual docker(MVD 2008.2.4.0 Molegro). The crystal structure of the (1G54) complexed with (E7070) was uploaded from protein data bank PDB ${ }^{(25)}$.

\section{Preparation of small molecule}

Molecular modeling of the target compounds were built using ChemDraw Ultra version 8.0.3 and minimized their energy through Chem3D Ultra version 8.0.3/MOPAC, Jop Type: Minimum RMS Gradient of $0.010 \mathrm{kcal} / \mathrm{mol}$ and RMS distance of $0.1^{\circ} \mathrm{A}$ and saved as MDL MolFile $(* \cdot \mathrm{mol})^{(27)}$. Our compounds were introduced into the (1G54) binding site in accordance with the published crystal structures of (E7070) bound to kinase. 
Active compounds and hCAII (PDB code 1G54) was uploaded without water molecules. The binding sites of (1G54) were defined close to Zn-Metal ion (volume of approximately 76.8 A3), The-199, His-119, His-98 and His-96 residues. During docking the grid resolution was set to $0.3 \AA$, while the binding site radius was set to $9 \AA$. Scorings generated by MVD. In addition . Docking Score and the following MVD based scores were also calculated for each compound.

(1) MVD score: Scorings generated by MVD.

(2) ReRank:To further increase the docking accuracy, the 10 solutions obtained from the 10 independent docking runs.

(3) T-interaction-E: total interaction energy between ligand and target.

(4) H. Bond: the hydrogen bonding energy between ligand and target.

(5) LE1: ligand efficiency 1, which corresponds to MolDock Scores divided by the number of heavy atoms.

(6) LE2: ligand efficiency 2, which corresponds to the affinity divided by the number of heavy atoms.

\section{References}

1. Rees, W., Keller, R., Vesenka, J., Yang, G. and Bustamante, C., Vidence of DNA bending in transcription complexes imaged by scanning force microscopy. Science, 260, 1646 (1993).

2. Wang, X. and Schneider, H., Binding of dansylamide derivatives to nucleotides and nucleic acids 1. J. Chem. Soc., Perkin Trans. 2, 1323(1998).

3. Sartorius, J. and Schneider, H., NMR-titrations with complexes between ds-DNA and indole derivatives including tryptophane containing peptides. FEBS Letter, 374, 387(1995).

4. Stewart, K. and Gray, Survey of the DNA binding properties of natural and synthetic polyamino compounds. T. J. Phys. Org. Chem. 5, 461(1992).

5. Gershon, H., Ghirlando, R., Guttman, S. and Minsky, A., Mode of formation and structural features of DNA-cationic liposome complexes used for transfection. Biochemistry, 32, 7143(1993).

6. El-Bendary, E. and Badria, F., Synthesis, DNA-binding, and antiviral activity of certain pyrazolo[3,4-d]pyrimidine derivatives. Arch. Pharm. Med. Chem. 333, 99(2000).

7. Yalçın, F., Biological activities of the marine sponge axinella Hacettepe University Journal of the Faculty of Pharmacy; 27(1), 47(2007).

Egypt. J. Chem. 53, No.2 (2010) 
8. Stanchev, S., Hadjimitova, V., Traykov, T., Boyanov, T. and Manolov, I., Investigation of the antioxidant properties of some new 4-hydroxycoumarin derivatives. Eur. J. Med Chem. 44, 3077(2009).

9. Zhang, W., Krohn, K., Draeger, S. and Schulz, B., Hualyzin, a symmetrical urea derivative isolated from penicillium herquei isolate GA4. J. Nat Prod. 71(6),1078(2008).

10. Hassan, G., Farag, N., Hegazy, G. and Arafa, R., Design and synthesis of novel benzopyran, 2-one derivatives of expected antimicrobial activity through dna gyrase-b inhibition. Arch Pharm (Weinheim). 341(11),725(2008),

11. Stasi, D., Camuesco, L., Nieto, D., Vilegas, A., Zarzuelo, W. and Galvez, A., Nestinal anti-inflammatory activity of paepalantine, an isocoumarin isolated from the capitula of paepalanthus bromelioides, in the trinitrobenzenesulphonic acid model of rat colitis. J. Planta Med. 70(4), 315(2004).

12. Heynekamp, J., Hunsaker, J., Jagt, T., Deck, L. and Jagt, D., Uncharged isocoumarin-based inhibitors of urokinase-type plasminogen activator. BMC Chem. Biol. 6, 3(2006).

13. Heynekamp, J., Hunsaker, L., Jagt, T., Royer, R. and Deck, L., Isocoumarinbased inhibitors of pancreatic cholesterol esterase. Bio.Org Med Chem. 16(9), 5285(2008).

14. Angelis, D., Stossi, F., Waibel, M., Katzene-llenbogen, B. and Katzenellenbogen J., Isocoumarins as estrogen receptor beta selective ligands: Isomers of isoflavone phytoestrogens and their metabolites. Bio.Org. Med. Chem. 13(23),6529(2005).

15. Checler, F., da-Costa, C., Ayral, E., Andrau, D., Dumanchin, C., Farzan, M., Hernandez, J., Martinez, J., Lefranc-Jullien, S., Maramb-aud, P., Pasini, A., Petit, A. , Phiel, C., Robert, P., George-Hyslop, P. and Wilk, S., Isocoumarin compounds as putative probes to selectively target the g-secretase pathway current alzheimer res., JLK Inhibitors, 2(3), 327(2005).

16. Jiun-Jie, S., Jim-Min, F., Chih-Jung, K., Tun-Hsun, K., Po-Huang, L., HungJyun, H., Wen-Bin, Y., Chun-Hung, L., Jiun-Ling, C., Yin-Ta , W. and ChiHuey, W., Discovery of potent anilide inhibitors against the severe acute respiratory syndrome 3cl protease. J. Med. Chem. 48, 4469(2005).

17. Hou, K., Wei, D., Zhong, W., Binding mechanism of coronavirus main proteinase with ligands and its implication to drug design against SARS Commun., Biochem. Bio. phys. Res. S 08, 148(2003).

18. Kivela, A.J., Kivela, J., Saarnio, J. and Parkkila, S., Loss of heterozygosity: An independent prognostic factor of colorectal cancer. World J. Gastroenterol. 11, 155(2005). 
19. Supuran, C.T., Carbonic anhydrases: novel therapeutic applications for inhibitors and activators. Nat. Rev. Drug Discov. 7, 168(2008).

20. Supuran, V. and Scozzafava, A., Supuran; carbonic anhydrases as targets for medicinal chemistry. Bioorg. Med. Chem. 15, 4336(2007).

21. Supuran, C.T., Scozzafava, A. and Casini, A., Carbonic anhydrase inhibitors. Med. Res. Rev. 23, 146(2003).

22. Casini, A., Scozzafava, A., Mastrolorenzo, and Supuran, L.T., Sulfonamides and sulfonylated derivatives as anticancer agents. Curr. Cancer Drug Targets, 2, 55(2002).

23. Pezzuto, J., Che, C., McPherson, D., Zhu, P., Topcu, G., Erdelmeier, C. and Cordell, G., DNA as an affinity probe useful in the detection and isolation of biologically active natural products. J. Nat. Prod. 54,1522(1991).

24. Burres, N., Frigo, A., Rasmussen, R. and McAlpine, J., A colorimetric microassay for the detection of agents that interact with DNA. J. Nat. Prod. 55, 1582(1992).

25. Thomsen, R. and Christensen, H., A new technique for high-accuracy molecular docking. J. Med. Chem. 49(11), 3315(2006),

26. Kim, C., Chang, J., Doyon, J., Baird, T., Fierke, C., Jain, A. and Christianson , D., Fluoroaromatic-fluoroaromatic interactions between inhibitors bound in the crystal lattice of human carbonic anhydrase II. Am.Chem.Soc. 122, 12125(2000).

27. ChemDraw Software [Available from: http://www.cambridgesoft.com/ software / ChemDraw/].lliams, Pharm. Biol. 32, 337-345(1994) .

(Received 29/3/2010 ; accepted 5/ 4/ 2010) 


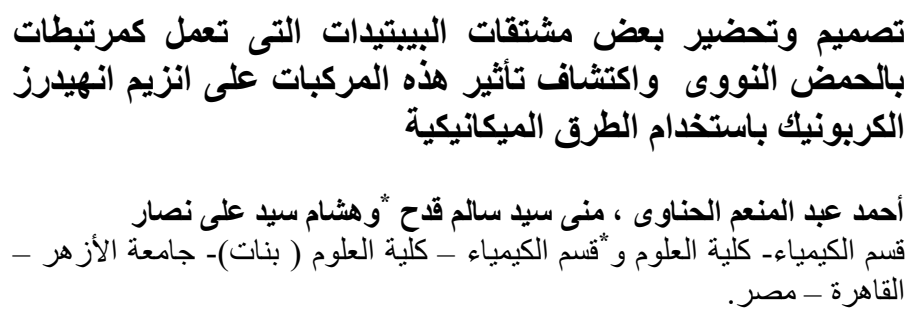

تحتوي هذه المقالة على وصف لعملية التشييد الكيميائي لمجموعة جديدة مشتقات

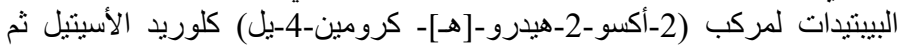

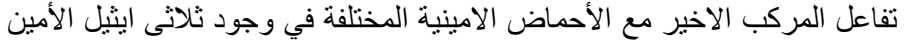

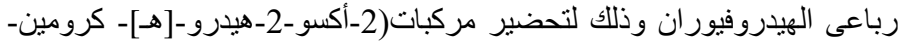

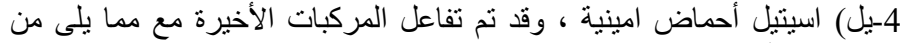

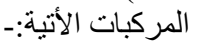

أـ كلوريد الثيونيل و الكحول الميثيلى لتحضير الاستر ات الميثيلية المقابلة.

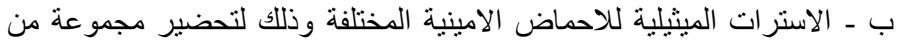

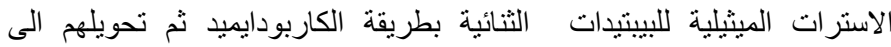

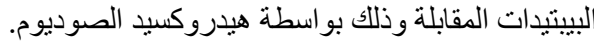

كما تم التأكد من التركيب البنائي لهذه المركبات الجديدة بواسطة التحليل

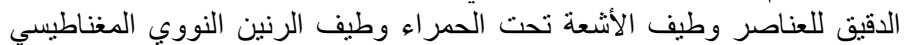

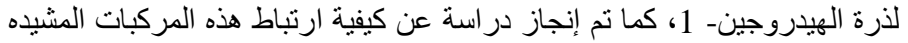

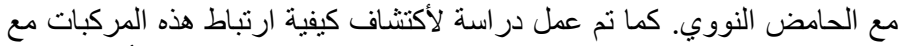

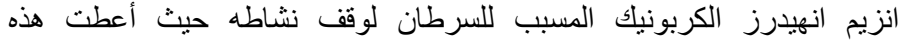

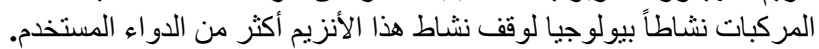

